\title{
Activity Confidence and Eating; a pilot group approach to management of childhood overweight and obesity: dietetic perspective in an interdisciplinary team
}

\author{
N. O'Keeffe ${ }^{1}$, E. Purtill ${ }^{1}$, M. Hegarty ${ }^{2}$ and C. Glennon ${ }^{1}$ \\ ${ }^{1}$ Community Nutrition and Dietetic Service, Health Service Executive Dublin Mid Leinster, Unit 25 Kilminchy Village, \\ Dublin Road, Portlaoise, Co. Laois, Republic of Ireland and ${ }^{2}$ Department of Public Health, Health Service Executive Dublin \\ Mid Leinster, Central Office, Arden Road, Tullamore, Co. Offaly, Republic of Ireland
}

Childhood obesity is a growing problem in Ireland, with $24 \%$ of children (aged 5-12 years) overweight or obese ${ }^{(1)}$. There is a need for effective interventions to treat these children. A group approach to treatment involving an interdisciplinary team has been recommended $^{(2)}$. The aim of the present study was to develop and implement the dietetic component for a pilot programme for the treatment of childhood overweight and obesity and evaluate anthropometric and dietary changes post intervention.

The Activity, Confidence and Eating (ACE) programme is a 12-week programme developed by an interdisciplinary working group including a dietitian, a psychologist and a physical activity health promotion officer. Eligibility criteria are children between the ages of 6 and 12 years with BMI above the 91st centile, with no medical cause for overweight or obesity. The dietetic component includes two education sessions with parents, one nutrition activity session with children and an education session with children and parents. Topics covered include healthy eating principles, portion sizes, snacks and healthy fluids. BMI $\left(\mathrm{kg} / \mathrm{m}^{2}\right)$ and waist circumference (cm) measurements were taken at baseline and repeated at 12 weeks (on completion of the intervention), 6 months and 12 months. A $3 \mathrm{~d}$ food diary measured change in energy intake. A semi-qualitative evaluation questionnaire was administered to parents post intervention.

Of the participants $92 \%$ (eleven of twelve) completed the 12-week programme. Anthropometric data for all time points were available for seven of eleven participants and are shown in the Table. Data for four of eleven participants were not available as they did not complete appointments at all data-collection points.

Table. Anthropometric changes for seven participants of the ACE programme at baseline, 12 weeks (on completion of the intervention), 6 months and 12 months

\begin{tabular}{|c|c|c|c|c|c|c|c|c|}
\hline \multirow[b]{2}{*}{ Measurement } & \multicolumn{2}{|c|}{ Baseline } & \multicolumn{2}{|c|}{12 weeks } & \multicolumn{2}{|c|}{6 months } & \multicolumn{2}{|c|}{12 months } \\
\hline & Mean & Range & Mean & Range & Mean & Range & Mean & Range \\
\hline BMI $\left(\mathrm{kg} / \mathrm{m}^{2}\right)$ & 29.5 & $24.8-40.3$ & 29.0 & $24.8-40.5$ & 29.2 & $23.7-42.3$ & 30.0 & $24.1-42.2$ \\
\hline Waist circumference $(\mathrm{cm})$ & 89.7 & $79-122$ & 89.8 & $79-121$ & 90.3 & $81-119$ & 94.9 & $86.5-126$ \\
\hline
\end{tabular}

Mean total energy intake were decreased at 12 weeks from $6720 \mathrm{~kJ}(n$ 9) to $6154 \mathrm{~kJ}(n 6)$; however, there was an increase at 6 months to $6682 \mathrm{~kJ}(n$ 6), with a slight decrease at 12 months to $6594 \mathrm{~kJ}(n$ 8). At 12 months all mean total energy intakes remained below the baseline mean intake. Nutritional knowledge increased and family changes occurred. Of the parents $64 \%$ (seven of eleven) completed questionnaires, and of these $100 \%$ rated the nutrition education session as 'good/very good' or 'excellent'. All seven respondents thought that their children had benefited from taking part in the project and would recommend it to other parents of overweight children. Comments from the facilitators included: 'The best aspects included the collaboration between disciplines but also collaboration with parents'.

The ACE programme was effective in decreasing BMI in the short term; however, long-term evaluation showed weight and waist circumference increased gradually post intervention. This finding suggests that families may require more intensive follow up, which should be taken into account when planning future programmes. Overall, the programme was well accepted by participants and parents and positive dietary changes were reported.

1. Irish Universities Nutrition Alliance (2005) National Children's Food Survey. http://www.iuna.net/childrens_survey/ (accessed April 2008).

2. Bauer B \& Maffeis C (2002) In Child and Adolescent Obesity, Causes and Consequences, Prevention and Management, pp. 361-376 [W Burniat, T Cole, I Lissau and E Poskitt, editors]. Cambridge: Cambridge University Press. 